\title{
Flavor-energy uncertainty relations for neutrino oscillations in quantum field theory
}

\author{
Massimo Blasone, ${ }^{1, *}$ Petr Jizba, ${ }^{2,3, \dagger}$ and Luca Smaldone ${ }^{1, *}$ \\ ${ }^{1}$ Dipartimento di Fisica, Università di Salerno, Via Giovanni Paolo II, 13284084 Fisciano, \\ Italy \& INFN Sezione di Napoli, Gruppo collegato di Salerno, Italy \\ ${ }^{2}$ FNSPE, Czech Technical University in Prague, Břehová 7, 11519 Praha 1, Czech Republic \\ ${ }^{3}$ ITP, Freie Universität Berlin, Arnimallee 14, D-14195 Berlin, Germany
}

(Received 8 October 2018; published 24 January 2019)

\begin{abstract}
In the context of quantum field theory, we derive flavor-energy uncertainty relations for neutrino oscillations. By identifying the nonconserved flavor charges with the "clock observables," we arrive at the Mandelstam-Tamm version of time-energy uncertainty relations. In the ultrarelativistic limit, these relations yield the well-known condition for neutrino oscillations. Ensuing nonrelativistic corrections to the latter are explicitly evaluated. The analogy among flavor states and unstable particles and a novel interpretation of our uncertainty relations, based on the unitary inequivalence of Fock spaces for flavor and massive neutrinos, are also discussed.
\end{abstract}

DOI: 10.1103/PhysRevD.99.016014

\section{INTRODUCTION}

Neutrino mixing and oscillations represent one of the most pressing challenges of modern theoretical and experimental particle physics. They were first introduced by Pontecorvo [1] in a close analogy with the phenomenon of Kaon oscillations [2], and subsequently confirmed in a number of experimental settings [3]. While the quantum mechanical (QM) description [4-6] is quite successful in tackling high-energy features of neutrino oscillations, the corresponding quantum field theoretical (QFT) description (which could tackle also the low-energy behavior) is still controversial [6-9].

In particular, a nonperturbative study of flavor states shows that field mixing is not the same as the wavefunction (i.e., first-quantized) mixing [8]. In fact, the flavor vacuum is structurally a condensate similar to the BCS vacuum $[8,10]$. From this, the corrections to the standard neutrino oscillation formula can be derived [11]. It should be stressed that in this analysis a central rôle is played by nonconserved flavor-charges implied by the Noether's theorem [12]. Flavor states are defined as eigenstates of such charges and have the form of $S U(2)$ generalized coherent states [13]. In fact, according to the standard

\footnotetext{
*blasone@sa.infn.it

†.jizba@fjfi.cvut.cz

"1smaldone@sa.infn.it
}

Published by the American Physical Society under the terms of the Creative Commons Attribution 4.0 International license. Further distribution of this work must maintain attribution to the author(s) and the published article's title, journal citation, and DOI. Funded by SCOAP. model (SM), the flavor charge is always conserved-at tree level - in the production and detection processes, a feature which is violated by the usual Pontecorvo flavor states [14]. In addition, the exact flavor states cannot be generally phrased as a simple superposition of mass eigenstates, because of the unitary inequivalence of mass and flavor Hilbert spaces [8].

In Ref. [15], it was shown that for neutrino oscillations described in terms of Pontecorvo states, the MandelstamTamm time-energy uncertainty relations (TEUR) [16] reduce to the known condition for neutrino oscillations [4]. However, this result was obtained in the context of standard perturbative treatment of neutrino flavor states.

In this paper we employ the full QFT framework to derive the flavor-energy uncertainty relations (FEUR). By identifying the nonconserved flavor charge with the "clock observable," we find from the latter the Mandelstam-Tamm version of TEUR. Our approach is valid at all energy scales and the conventional results of Bilenky et al. [15] are recovered in the ultrarelativistic limit. Moreover, by exploiting the analogy between flavor neutrinos and unstable particles [17], we find that for an exact neutrino flavor state, an inherent energy uncertainty arises from TEUR. Although our discussion is, for simplicity's sake, confined to two flavors only, the results obtained can be easily extended to three flavors including $C P$ violation.

\section{NEUTRINO MIXING AND OSCILLATIONS IN QFT}

Let us consider a weak decay $W^{+} \rightarrow e^{+}+\nu_{e}$. The relevant part of the SM Lagrangian is $\mathcal{L}=\mathcal{L}_{0}+\mathcal{L}_{\text {int }}$ with 


$$
\begin{aligned}
\mathcal{L}_{0} & =\bar{\nu}\left(i \gamma_{\mu} \partial^{\mu}-M_{\nu}\right) \nu+\bar{l}\left(i \gamma_{\mu} \partial^{\mu}-M_{l}\right) l, \\
\mathcal{L}_{\text {int }} & =\frac{g}{2 \sqrt{2}}\left[W_{\mu}^{+} \bar{\nu} \gamma^{\mu}\left(1-\gamma^{5}\right) l+\text { H.c. }\right],
\end{aligned}
$$

where $\nu=\left(\nu_{e}, \nu_{\mu}\right)^{T}, l=(e, \mu)^{T}$, and

$$
M_{\nu}=\left(\begin{array}{cc}
m_{e} & m_{e \mu} \\
m_{e \mu} & m_{\mu}
\end{array}\right), \quad M_{l}=\left(\begin{array}{cc}
\tilde{m}_{e} & 0 \\
0 & \tilde{m}_{\mu}
\end{array}\right) .
$$

The Lagrangian $\mathcal{L}$ is invariant under the global $U(1)$ transformations $\nu \rightarrow e^{i \alpha} \nu$ and $l \rightarrow e^{i \alpha} l$, leading to the conservation of the total flavor charge $Q_{l}^{\text {tot }}$ corresponding to the lepton-number conservation [5]. This can be written in terms of the flavor charges for neutrinos and charged leptons [12],

$$
Q_{l}^{\mathrm{tot}}=\sum_{\sigma=e, \mu} Q_{\sigma}^{\mathrm{tot}}(t), \quad Q_{\sigma}^{\mathrm{tot}}(t)=Q_{\nu_{\sigma}}(t)+Q_{\sigma}(t),
$$

with

$$
\begin{aligned}
Q_{e} & =\int \mathrm{d}^{3} x e^{\dagger}(x) e(x), & Q_{\nu_{e}}(t) & =\int \mathrm{d}^{3} x \nu_{e}^{\dagger}(x) \nu_{e}(x), \\
Q_{\mu} & =\int \mathrm{d}^{3} x \mu^{\dagger}(x) \mu(x), & Q_{\nu_{\mu}}(t) & =\int \mathrm{d}^{3} x \nu_{\mu}^{\dagger}(x) \nu_{\mu}(x) .
\end{aligned}
$$

The above charges can be derived via Noether's theorem [12] from the Lagrangian (1). Note the time dependence of the neutrino charges, due to the nondiagonal mass matrix $M_{\nu}$.

By observing that $\left[\mathcal{L}_{\text {int }}(\mathbf{x}, t), Q_{\sigma}^{\text {tot }}(t)\right]=0$, we see that a neutrino flavor state is well defined in the production vertex as an eigenstate of the corresponding flavor charge [18].

The mixing relations for neutrino fields are

$$
\left(\begin{array}{l}
\nu_{e}(x) \\
\nu_{\mu}(x)
\end{array}\right)=\left(\begin{array}{cc}
\cos \theta & \sin \theta \\
-\sin \theta & \cos \theta
\end{array}\right)\left(\begin{array}{l}
\nu_{1}(x) \\
\nu_{2}(x)
\end{array}\right),
$$

with $\tan 2 \theta=2 m_{e \mu} /\left(m_{\mu}-m_{e}\right)$. The fields with definite masses have the usual mode expansion,

$$
\begin{aligned}
\nu_{j}(x)= & \sum_{r} \int \frac{\mathrm{d}^{3} k}{(2 \pi)^{\frac{3}{2}}}\left[u_{\mathbf{k}, j}^{r}(t) \alpha_{\mathbf{k}, j}^{r}(t)\right. \\
& \left.+v_{-\mathbf{k}, j}^{r}(t) \beta_{-\mathbf{k}, j}^{r \dagger}(t)\right] e^{i \mathbf{k} \cdot \mathbf{x}}, \quad j=1,2,
\end{aligned}
$$

where

$$
\left(\begin{array}{l}
m_{e} \\
m_{\mu}
\end{array}\right)=\left(\begin{array}{cc}
\cos ^{2} \theta & \sin ^{2} \theta \\
\sin ^{2} \theta & \cos ^{2} \theta
\end{array}\right)\left(\begin{array}{l}
m_{1} \\
m_{2}
\end{array}\right)
$$

Equation (5) can be equivalently rewritten as [8]

$$
\nu_{\sigma}(x)=G_{\theta}^{-1}(t) \nu_{j}(x) G_{\theta}(t),
$$

with $(\sigma, j)=(e, 1),(\mu, 2)$ and $G_{\theta}(t)$ given by

$G_{\theta}(t)=\exp \left[\theta \int \mathrm{d}^{3} x\left(\nu_{1}^{\dagger}(x) \nu_{2}(x)-\nu_{2}^{\dagger}(x) \nu_{1}(x)\right)\right]$.

From (6) and (8), it follows that the flavor fields are

$$
\begin{aligned}
\nu_{\sigma}(x)= & \sum_{r} \int \frac{\mathrm{d}^{3} k}{(2 \pi)^{\frac{3}{2}}}\left[u_{\mathbf{k}, j}^{r}(t) \alpha_{\mathbf{k}, \sigma}^{r}(t)\right. \\
& \left.+v_{-\mathbf{k}, j}^{r}(t) \beta_{-\mathbf{k}, \sigma}^{r \dagger}(t)\right] e^{i \mathbf{k} \cdot \mathbf{x}},
\end{aligned}
$$

with $(\sigma, j)=(e, 1),(\mu, 2)$, and flavor ladder operators given by [19]

$$
\left(\begin{array}{c}
\alpha_{\mathbf{k}, \sigma}^{r}(t) \\
\beta_{-\mathbf{k}, \sigma}^{r}(t)
\end{array}\right)=G_{\theta}^{-1}(t)\left(\begin{array}{c}
\alpha_{\mathbf{k}, j}^{r}(t) \\
\beta_{-\mathbf{k}, j}^{r}(t)
\end{array}\right) G_{\theta}(t) .
$$

The vacuum for massive fields, $|0\rangle_{1,2}$, is defined as

$$
\alpha_{\mathbf{k}, j}^{r}|0\rangle_{1,2}=\beta_{-\mathbf{k}, j}^{r}|0\rangle_{1,2}=0 \quad j=1,2
$$

and is not left-invariant under the action of $G_{\theta}(t)$ :

$$
|0(t)\rangle_{e, \mu}=G_{\theta}^{-1}(t)|0\rangle_{1,2} .
$$

$|0(t)\rangle_{e, \mu}$ is called the flavor vacuum, and one can easily verify that it is annihilated by the flavor operators defined in Eq. (11). Moreover, one can prove [8] that

$\lim _{V \rightarrow \infty}\langle 0 \mid 0(t)\rangle_{e, \mu}=\lim _{V \rightarrow \infty} e^{\frac{V}{(2 \pi)^{3}} \int \mathrm{d}^{3} k \ln \left(1-\sin ^{2} \theta\left|V_{\mathbf{k}}\right|^{2}\right)^{2}}=0$,

where

$\left|V_{\mathbf{k}}\right|=\sqrt{\frac{|\mathbf{k}|}{4 \omega_{\mathbf{k}, 1} \omega_{\mathbf{k}, 1}}}\left(\sqrt{\frac{\omega_{\mathbf{k}, 2}+m_{2}}{\omega_{\mathbf{k}, 1}+m_{1}}}-\sqrt{\frac{\omega_{\mathbf{k}, 1}+m_{1}}{\omega_{\mathbf{k}, 2}+m_{2}}}\right)$,

i.e., flavor and massive fields belong to unitarily inequivalent representations of the anticommutation relations [8].

\section{MANDELSTAM-TAMM TEUR}

The Mandelstam-Tamm version of TEUR is formulated as [16]

$$
\Delta E \Delta t \geq \frac{1}{2}
$$

where

$$
\Delta E \equiv \sigma_{H} \quad \Delta t \equiv \sigma_{O} /\left|\frac{\mathrm{d}\langle O(t)\rangle}{\mathrm{d} t}\right|
$$


Here, $O(t)$ represents the "clock observable" whose dynamics quantifies temporal changes in a system, and $\Delta t$ is the characteristic time interval over which the mean value of $O$ changes by a standard deviation.

TEUR (16)-(17) is typically applied to the study of unstable particles; see, e.g., [20]. Calling $|\phi(t)\rangle$ an eigenstate of the projection operator $P_{\phi}(t)=|\phi(t)\rangle\langle\phi(t)|$ describing an unstable particle state, one gets [17]

$$
\left|\frac{\mathrm{d} \mathcal{P}_{\phi}(t)}{\mathrm{d} t}\right| \leq 2 \Delta E \sqrt{\mathcal{P}_{\phi}(t)\left(1-\mathcal{P}_{\phi}(t)\right)} .
$$

Here, $\mathcal{P}_{\phi}(t)$ is the survival probability

$$
\mathcal{P}_{\phi}(t)=|\langle\phi(t) \mid \phi\rangle|^{2},
$$

and $|\phi\rangle$ is the (Heisenberg representation) state of the system prepared at $t=0$.

The rhs of (18) has a maximum when $\mathcal{P}_{\phi}(t)=\frac{1}{2}$, which is satisfied with $t=T_{h}$. Thus, we have

$$
\Delta E \geq\left|\frac{\mathrm{d} \mathcal{P}_{\phi}(t)}{\mathrm{d} t}\right|
$$

Because for decaying particles $\mathcal{P}_{\phi}(0)=1, \mathcal{P}_{\phi}(\infty)=0$, and $\mathcal{P}_{\phi}(t)$ is monotonically decreasing, we can integrate the inequality (18), obtaining

$$
\Delta E T \geq \frac{1}{2}\left[\frac{\pi}{2}-\arcsin \left(2 \mathcal{P}_{\phi}(T)-1\right)\right] .
$$

From this, one can derive an explicit form of TEUR for unstable particles [17]

$$
\Delta E T_{h} \geq \frac{\pi}{4} .
$$

One can use a similar line of reasonings to arrive at the TEUR for the neutrino oscillations [15]. However, in order to remain as close as possible to the full QFT treatment, we employ a different strategy. We start by considering the number operator for flavor neutrinos:

$$
\tilde{N}_{\sigma}(t)=\sum_{\mathbf{k}, r} \tilde{\alpha}_{\mathbf{k}, \sigma}^{r \dagger}(t) \tilde{\alpha}_{\mathbf{k}, \sigma}^{r}(t), \quad \sigma=e, \mu,
$$

where

$$
\left(\begin{array}{c}
\tilde{\alpha}_{\mathbf{k}, e}^{r} \\
\tilde{\alpha}_{\mathbf{k}, \mu}^{r}
\end{array}\right) \equiv\left(\begin{array}{cc}
\cos \theta & \sin \theta \\
-\sin \theta & \cos \theta
\end{array}\right)\left(\begin{array}{c}
\alpha_{\mathbf{k}, 1}^{r} \\
\alpha_{\mathbf{k}, 2}^{r}
\end{array}\right)
$$

These relations are just approximations of the exact ones (11) in the ultrarelativistic limit. Defining the Pontecorvo flavor state as

$$
\left|\nu_{\mathbf{k}, \sigma}^{r}\right\rangle_{P} \equiv \tilde{\alpha}_{\mathbf{k}, \sigma}^{r \dagger}|0\rangle_{1,2}
$$

one gets the usual relations among flavor and massive neutrino states

$$
\left(\begin{array}{l}
\left|\nu_{\mathbf{k}, e}^{r}\right\rangle_{P} \\
\left|\nu_{\mathbf{k}, \mu}^{r}\right\rangle_{P}
\end{array}\right)=\left(\begin{array}{cc}
\cos \theta & \sin \theta \\
-\sin \theta & \cos \theta
\end{array}\right)\left(\begin{array}{c}
\left|\nu_{\mathbf{k}, 1}^{r}\right\rangle \\
\left|\nu_{\mathbf{k}, 2}^{r}\right\rangle
\end{array}\right) .
$$

The standard oscillation formula [1] can be found by taking the expectation value of the number operator over the corresponding Pontecorvo flavor state

$$
\mathcal{P}_{\sigma \rightarrow \sigma}(t)=\left\langle\tilde{N}_{\sigma}(t)\right\rangle_{\sigma}=1-\sin ^{2}(2 \theta) \sin ^{2}\left(\frac{\omega_{\mathbf{k}, 1}-\omega_{\mathbf{k}, 2}}{2} t\right),
$$

where $\langle\cdots\rangle_{\sigma}={ }_{P}\left\langle\nu_{\mathbf{k}, \sigma}^{r}|\cdots| \nu_{\mathbf{k}, \sigma}^{r}\right\rangle_{P}$. By setting $O(t)=\tilde{N}_{\sigma}(t)$ in (17) and taking into account that

$$
\begin{aligned}
\sigma_{N}^{2} & =\left\langle\tilde{N}_{\sigma}^{2}(t)\right\rangle_{\sigma}-\left\langle\tilde{N}_{\sigma}(t)\right\rangle_{\sigma}^{2} \\
& =\mathcal{P}_{\sigma \rightarrow \sigma}(t)\left(1-\mathcal{P}_{\sigma \rightarrow \sigma}(t)\right),
\end{aligned}
$$

one gets

$$
\left|\frac{\mathrm{d} \mathcal{P}_{\sigma \rightarrow \sigma}(t)}{\mathrm{d} t}\right| \leq 2 \Delta E \sqrt{\mathcal{P}_{\sigma \rightarrow \sigma}(t)\left(1-\mathcal{P}_{\sigma \rightarrow \sigma}(t)\right)} .
$$

This is formally identical to relation (18) for unstable particles. Note that $\sigma_{N}^{2}$ is proportional to the linear entropy which quantifies the dynamical flavor entanglement of the state (25), cf. [21]. Moreover, (29) is related to the WignerYanase skew-information [22] which reduces to the standard variance on pure states [23].

If we consider $\mathcal{P}_{\sigma \rightarrow \sigma}(t)$ in the interval $0 \leq t \leq t_{1 \mathrm{~min}}$, where $t_{1 \mathrm{~min}}$ is the time when $\mathcal{P}_{\sigma \rightarrow \sigma}(t)$ reaches the first minimum, this is a monotonically decreasing function [15]. In other words, if we try to reveal neutrinos in processes with time scales much smaller than oscillation time, they can be thought as unstable particles. In fact, in this time interval, we can regain, by integration, an expression analogous to (21).

However, a general inequality, not restricted to a particular time interval, can also be obtained from (20). Using the triangular inequality and integrating both sides from 0 to $T$, we get

$$
\Delta E T \geq \int_{0}^{T} \mathrm{~d} t\left|\frac{\mathrm{d} \mathcal{P}_{\sigma \rightarrow \sigma}(t)}{\mathrm{d} t}\right| \geq\left|\int_{0}^{T} \mathrm{~d} t \frac{\mathrm{d} \mathcal{P}_{\sigma \rightarrow \sigma}(t)}{\mathrm{d} t}\right| .
$$

Therefore, one finds

$$
\Delta E T \geq \mathcal{P}_{\sigma \rightarrow \rho}(T), \quad \sigma \neq \rho,
$$

with $\mathcal{P}_{\sigma \rightarrow \rho}(t)=1-\mathcal{P}_{\sigma \rightarrow \sigma}(t)$. For $T=T_{h}$, we finally have 


$$
\Delta E T_{h} \geq \frac{1}{2},
$$

which is even stronger than (22) and, in addition, it has Heisenberg-like lower bound.

\section{TEUR FOR NEUTRINO OSCILLATIONS IN QFT}

Let us now consider a full QFT treatment of TEUR. We have seen that these relations are a consequence of the nonconservation of the number of neutrinos with definite flavor. However, we used basically a quantum mechanical treatment, having approximated the flavor neutrino states with the simple expression (26). One can check that these are not eigenstates of the flavor charges (4). True flavor eigenstates can be explicitly constructed as

$$
\left|\nu_{\mathbf{k}, \sigma}^{r}\right\rangle=\alpha_{\mathbf{k}, \sigma}^{r \dagger}|0\rangle_{e, \mu}
$$

where flavor operators and vacuum are taken at reference time $t=0$. The corresponding oscillation formula can be found by taking the expectation value of the flavor charges [11],

$$
\mathcal{Q}_{\sigma \rightarrow \rho}(t)=\left\langle Q_{\nu_{\rho}}(t)\right\rangle_{\sigma}
$$

where $\langle\cdots\rangle_{\sigma}=\left\langle\nu_{\mathbf{k}, \sigma}^{r}|\cdots| \nu_{\mathbf{k}, \sigma}^{r}\right\rangle$, which gives

$$
\begin{aligned}
& \mathcal{Q}_{\sigma \rightarrow \rho}(t)=\sin ^{2}(2 \theta)\left[\left|U_{\mathbf{k}}\right|^{2} \sin ^{2}\left(\omega_{\mathbf{k}}^{-} t\right)+\left|V_{\mathbf{k}}\right|^{2} \sin ^{2}\left(\omega_{\mathbf{k}}^{+} t\right)\right] \\
& \mathcal{Q}_{\sigma \rightarrow \sigma}(t)=1-\mathcal{Q}_{\sigma \rightarrow \rho}(t), \quad \sigma \neq \rho
\end{aligned}
$$

where now $\omega_{\mathbf{k}}^{ \pm} \equiv\left(\omega_{\mathbf{k}, 2} \pm \omega_{\mathbf{k}, 1}\right) / 2$ and $\left|U_{\mathbf{k}}\right|^{2}=1-\left|V_{\mathbf{k}}\right|^{2}$. This formula presents oscillations on two different timescales: $T_{-}=2 \pi / \omega_{\mathbf{k}}^{-}$, which is the main one, observed also in the standard treatment, and $T_{+}=2 \pi / \omega_{\mathbf{k}}^{+}$, due to the interaction with the flavor vacuum condensate [8].

In analogy with the above QM treatment for the number operators, nonconservation of the flavor charges leads to a particular form of the QFT-based TEUR. This is because, in this case, lepton charge is a natural candidate for a "clock observable." By employing the fact that

$$
\left[Q_{\nu_{\sigma}}(t), H\right]=i \frac{\mathrm{d} Q_{\nu_{\sigma}}(t)}{\mathrm{d} t} \neq 0
$$

we find the flavor-energy uncertainty relation

$$
\sigma_{H} \sigma_{Q} \geq \frac{1}{2}\left|\frac{\mathrm{d} \mathcal{Q}_{\sigma \rightarrow \sigma}(t)}{\mathrm{d} t}\right|
$$

Moreover, one can verify that

$$
\begin{aligned}
\sigma_{Q}^{2} & =\left\langle Q_{\nu_{\sigma}}^{2}(t)\right\rangle_{\sigma}-\left\langle Q_{\nu_{\sigma}}(t)\right\rangle_{\sigma}^{2} \\
& =\mathcal{Q}_{\sigma \rightarrow \sigma}(t)\left(1-\mathcal{Q}_{\sigma \rightarrow \sigma}(t)\right)
\end{aligned}
$$

Equation (38) quantifies dynamical (flavor) entanglement for neutrino states in QFT, cf. Refs. [24,25]). This should be compared to results (18) and (29). By analogy with (20), one has

$$
\left|\frac{\mathrm{d} \mathcal{Q}_{\sigma \rightarrow \sigma}(t)}{\mathrm{d} t}\right| \leq \Delta E .
$$

From (37), we arrive at the Mandelstam-Tamm TEUR in the form

$$
\Delta E T \geq \mathcal{Q}_{\sigma \rightarrow \rho}(T), \quad \sigma \neq \rho .
$$

When $m_{i} /|\mathbf{k}| \rightarrow 0$, i.e., in the relativistic case, we get

$$
\left|U_{\mathbf{k}}\right|^{2} \approx 1-\varepsilon(\mathbf{k}), \quad\left|V_{\mathbf{k}}\right|^{2} \approx \varepsilon(\mathbf{k}),
$$

with $\varepsilon(\mathbf{k}) \equiv\left(m_{1}-m_{2}\right)^{2} / 4|\mathbf{k}|^{2}$. In the same limit,

$$
\omega_{\mathbf{k}}^{-} \approx \frac{\delta m^{2}}{4|\mathbf{k}|}=\frac{\pi}{L_{\mathrm{osc}}}, \quad \omega_{\mathbf{k}}^{+} \approx|\mathbf{k}|,
$$

where $\delta m^{2} \equiv m_{2}^{2}-m_{1}^{2}$ and $L_{\text {osc }} \equiv 4 \pi|\mathbf{k}| / \delta m^{2}$. Therefore, at the leading order (ultrarelativistic case) $\left|U_{\mathbf{k}}\right|^{2} \rightarrow 1$, $\left|V_{\mathbf{k}}\right|^{2} \rightarrow 0$. In this limit, the standard oscillation formula (27) is recovered,

$$
\mathcal{Q}_{\sigma \rightarrow \rho}(t) \approx \sin ^{2}(2 \theta) \sin ^{2}\left(\frac{\pi L}{L_{\mathrm{osc}}}\right), \quad \sigma \neq \rho,
$$

where we put $t \approx L$. The rhs of (43) reaches its maximum at $L=L_{\mathrm{osc}} / 2$ and the inequality (40) reads

$$
\Delta E \geq \frac{2 \sin ^{2}(2 \theta)}{L_{\mathrm{osc}}}
$$

Note that because the Hamiltonian is time independent, so is $\Delta E$. In particular, the relation (44) applies in the interaction vertex. Inequalities of the form (44) are well known in literature and are usually interpreted as conditions of neutrino oscillations $[4,7,15]$.

Having based our derivation on exact flavor states and charges, we can see the above relations in a new light: From the inequality (44), we infer that flavor neutrinos have an inherent energy uncertainty which represents a bound for future experiments. In order to clarify this statement, note that (14) implies that

$$
\lim _{V \rightarrow \infty}\left\langle\nu_{\mathbf{k}, i}^{r} \mid \nu_{\mathbf{k}, \sigma}^{r}\right\rangle=0, \quad i=1,2,
$$

i.e., the flavor neutrino state, which is produced in charged current weak decays, cannot be written as a linear 
superposition of single-particle mass eigenstates. The orthogonality condition (45) does not hold for the standard flavor states (26), where $\lim _{V \rightarrow \infty}\left\langle\nu_{\mathbf{k}, 1}^{r} \mid \nu_{\mathbf{k}, e}^{r}\right\rangle_{P}=\cos \theta$. This contradiction is resolved by observing that

$$
\lim _{m_{i} /|\mathbf{k}| \rightarrow 0} \lim _{V \rightarrow \infty} \neq \lim _{V \rightarrow \infty} \lim _{m_{i} /|\mathbf{k}| \rightarrow 0}
$$

which means that the ultrarelativistic limit cannot be taken once the "thermodynamical" QFT limit is performed, but has to be considered just as QM approximation, which does not hold for systems with an infinite number of degrees of freedom. Equation (45) should be thus understood as

$$
\left\langle\nu_{\mathbf{k}, i}^{r} \mid \nu_{\mathbf{k}, \sigma}^{r}\right\rangle={ }_{1,2}\left\langle 0_{\mathbf{k}}\left|\alpha_{\mathbf{k}, 1}^{r} \alpha_{\mathbf{k}, e}^{r \dagger}\right| 0_{\mathbf{k}}\right\rangle_{e, \mu} \prod_{\mathbf{p} \neq \mathbf{k}}\left\langle 0_{1,2} \mid 0_{\mathbf{p}}\right\rangle_{e, \mu} .
$$

Let us now consider corrections beyond the ultrarelativistic limit. The exact oscillation formula (35) reduces in the next-to-leading relativistic order to [26]

$$
\begin{aligned}
\mathcal{Q}_{\sigma \rightarrow \rho}(t) \approx & \sin ^{2}(2 \theta)\left[\sin ^{2}\left(\frac{\pi t}{L_{\text {osc }}}\right)(1-\varepsilon(\mathbf{k}))\right. \\
& \left.+\varepsilon(\mathbf{k}) \sin ^{2}(|\mathbf{k}| t)\right], \quad \sigma \neq \rho .
\end{aligned}
$$

By setting $T=L_{\mathrm{osc}} / 2$, the relation (40) can be written as

$$
\Delta E \geq \frac{2 \sin ^{2} 2 \theta}{L_{\mathrm{osc}}}\left[1-\varepsilon(\mathbf{k}) \cos ^{2}\left(\frac{|\mathbf{k}| L_{\mathrm{osc}}}{2}\right)\right]
$$

i.e., the bound on the energy is lowered with respect to (44). For neutrino masses [27]: $m_{1}=0.0497 \mathrm{eV}, m_{2}=$ $0.0504 \mathrm{eV}$, and $|\mathbf{k}|=1 \mathrm{MeV}$, then $\varepsilon(\mathbf{k})=2 \times 10^{-19}$.

On the other hand, in the nonrelativistic regime where the pure QFT effects (such as interactions with the vacuum) are relevant, the full oscillation formula simplifies. To this end, we consider, e.g., $|\mathbf{k}|=\sqrt{m_{1} m_{2}}$. In this case,

$$
\begin{gathered}
\left|U_{\mathbf{k}}\right|^{2}=\frac{1}{2}+\frac{\xi}{2}=1-\left|V_{\mathbf{k}}\right|^{2}, \\
\xi=\frac{2 \sqrt{m_{1} m_{2}}}{m_{1}+m_{2}},
\end{gathered}
$$

and we can rewrite (40) as

$$
\begin{aligned}
\Delta E T \geq & \frac{\sin ^{2} 2 \theta}{2}\left[1-\cos \left(\tilde{\omega}_{1} T\right) \cos \left(\tilde{\omega}_{2} T\right)\right. \\
& \left.-\xi \sin \left(\tilde{\omega}_{1} T\right) \sin \left(\tilde{\omega}_{2} T\right)\right],
\end{aligned}
$$

with $\tilde{\omega}_{j}=\sqrt{m_{j}\left(m_{1}+m_{2}\right)}$. To compare it with the ultrarelativistic case, we take $T=\tilde{L}_{\text {osc }} / 4$, with $\tilde{L}_{\text {osc }}=$ $4 \pi \sqrt{m_{1} m_{2}} / \delta m^{2}$, obtaining

$$
\Delta E \geq \frac{2 \sin ^{2} 2 \theta}{\tilde{L}_{\mathrm{osc}}}(1-\chi)
$$

Here,

$$
\begin{aligned}
\chi= & \xi \sin \left(\tilde{\omega}_{1} \tilde{L}_{\mathrm{osc}} / 4\right) \sin \left(\tilde{\omega}_{2} \tilde{L}_{\mathrm{osc}} / 4\right) \\
& +\cos \left(\tilde{\omega}_{1} \tilde{L}_{\mathrm{osc}} / 4\right) \cos \left(\tilde{\omega}_{2} \tilde{L}_{\mathrm{osc}} / 4\right) .
\end{aligned}
$$

Substituting the same values as above, for neutrino masses, we obtain $\chi=0.1$, i.e., the original bound on energy decreased by $10 \%$.

\section{CONCLUSIONS}

By identifying the flavor charges obtained via Noether's theorem and energy as incompatible observables, we have derived flavor-energy uncertainty relations. Taking the nonconserved flavor charges as "clock observables," we arrived at the Mandelstam-Tamm version of TEUR, in a full QFT framework. In the ultrarelativistic regime, our results reproduce the standard conditions for neutrino oscillations from Refs. [4,7,15], thus incorporating the achievements of Ref. [15].

Unlike Ref. [15], our result is valid for all times and energy scales and improves the bounds of Ref. [17]. We have interpreted TEUR for flavor neutrinos as representing fundamental bounds on energy-variances. This interpretation is drawn in a close analogy with the case of unstable particles, where a notion of a sharp mass is not natural, and only mass (energy) distributions are measurable.

We would like to stress that the reason why the results obtained here generalize, in a natural way, the usual QM results resides in the fact that the QFT flavor neutrino states are defined as eigenstates of the flavor charges. This is a nontrivial step which is possible because of the unitary inequivalence of the Hilbert spaces for neutrinos with definite masses and those with definite flavor [8].

Our study naturally correlates with the research line of Refs. [21,25,29] where neutrino oscillations have been studied from a quantum information perspective. Finally, we note that analogous analysis can be applied to boson mixing, such as for $K^{0}, D^{0}$, or $B^{0}$ mesons.

\section{ACKNOWLEDGMENTS}

P. J. acknowledges support from the Czech Science Foundation (GAČR), Grant No. 17-33812L. 
[1] B. Pontecorvo, Sov. Phys. JETP 6, 429 (1957); 7, 172 (1957); V. Gribov and B. Pontecorvo, Phys. Lett. 28B, 493 (1969); S. M. Bilenky and B. Pontecorvo, Phys. Lett. 61B, 248 (1976); Phys. Rep. 41, 225 (1978).

[2] M. Gell Mann and A. Pais, Phys. Rev. 97, 1387 (1955).

[3] W. Hampel et al. (GALLEX Collaboration), Phys. Lett. B 447, 127 (1999); M. Altmann et al. (GNO Collaboration), Phys. Lett. B 490, 16 (2000); M. B. Smy (Super-Kamiokande Collaboration), Nucl. Phys. B, Proc. Suppl. 118, 25 (2003); A. L. Hallin et al., Nucl. Phys. B, Proc. Suppl. 118, 3 (2003); K. Eguchi et al. (KamLAND Collaboration), Phys. Rev. Lett. 90, 021802 (2003).

[4] B. Kayser, Phys. Rev. D 24, 110 (1981).

[5] S. M. Bilenky and S. T. Petcov, Rev. Mod. Phys. 59, 671 (1987).

[6] C. Giunti and C. W. Kim, Fundamentals of Neutrino Physics and Astrophysics (Oxford University, New York, 2007).

[7] W. Grimus and P. Stockinger, Phys. Rev. D 54, 3414 (1996); A. G. Cohen, S. L. Glashow, and Z. Ligeti, Phys. Lett. B 678, 191 (2009); E. K. Akhmedov and A. Y. Smirnov, Phys. At. Nucl. 72, 1363 (2009).

[8] M. Blasone and G. Vitiello, Ann. Phys. (N.Y.) 244, 283 (1995).

[9] K. Fujii, C. Habe, and T. Yabuki, Phys. Rev. D 64, 013011 (2001); C. R. Ji and Y. Mishchenko, Phys. Rev. D 65, 096015 (2002); K. C. Hannabuss and D. C. Latimer, J. Phys. A 36, L69 (2003).

[10] L. N. Chang and N. P. Chang, Phys. Rev. Lett. 45, 1540 (1980).

[11] M. Blasone, P. A. Henning, and G. Vitiello, Phys. Lett. B 451, 140 (1999); M. Blasone, P. Pires Pacheco, and H. W. C. Tseung, Phys. Rev. D 67, 073011 (2003).

[12] M. Blasone, P. Jizba, and G. Vitiello, Phys. Lett. B 517, 471 (2001).

[13] A. Perelomov, Generalized Coherent States and their Applications (Springer-Verlag, Berlin, 1986).
[14] M. Blasone, A. Capolupo, C. R. Ji, and G. Vitiello, Int. J. Mod. Phys. A 25, 4179 (2010).

[15] S. M. Bilenky, Phys. Scr. T T127, 8 (2006); S. M. Bilenky and M. D. Mateev, Phys. Part. Nucl. 38, 117 (2007); S. M. Bilenky, F. von Feilitzsch, and W. Potzel, J. Phys. G 35, 095003 (2008); S. M. Bilenky, F. von Feilitzsch, and W. Potzel, J. Phys. G 38, 115002 (2011).

[16] L. Mandelstam and I. G. Tamm, J. Phys. (Moscow) 9, 249 (1945).

[17] K. Bhattacharyya, J. Phys. A 16, 2993 (1983).

[18] M. Blasone, A. Capolupo, C. R. Ji, and G. Vitiello, Nucl. Phys. B, Proc. Suppl. 188, 37 (2009).

[19] Here we choose a Lorentz frame so that $\mathbf{k}=(0,0,|\mathbf{k}|)$.

[20] D. J. Griffiths, Introduction to Quantum Mechanics (Prentice Hall, New Jersey, 1995); B. Schumacher and M. Westmoreland, Quantum Processes, Systems, and Information (Cambridge University Press, Cambridge, England, 2010).

[21] M. Blasone, F. Dell'Anno, S. De Siena, and F. Illuminati, Europhys. Lett. 85, 50002 (2009); Nucl. Phys. B, Proc. Suppl. 237-238, 320 (2013).

[22] E. P. Wigner and M. M. Yanase, Proc. Natl. Acad. Sci. U.S.A. 49, 910 (1963).

[23] S. Luo, Phys. Rev. Lett. 91, 180403 (2003).

[24] A. A. Klyachko, B. Öztop, and A. S. Shumovsky, Phys. Rev. A 75, 032315 (2007).

[25] M. Blasone, F. Dell'Anno, S. De Siena, and F. Illuminati, J. Phys. Conf. Ser. 442, 012070 (2013); Europhys. Lett. 112, 20007 (2015).

[26] C. Y Lee, arXiv:1709.06306.

[27] This values for neutrino masses are taken from [28], in the case of inverted hierarchy.

[28] M. Tanabashi et al. (Particle Data Group), Phys. Rev. D 98, 030001 (2018).

[29] S. Banerjee, A. K. Alok, R. Srikanth, and B. C. Hiesmayr, Eur. Phys. J. C 75, 487 (2015); A. K. Alok, S. Banerjee, and S. U. Sankar, Nucl. Phys. B909, 65 (2016). 